\title{
Trichostatin A potentiates genistein-induced apoptosis and reverses EMT in HEp2 cells
}

\author{
RUIXIA DU ${ }^{1,2}$, ZHE LIU ${ }^{3}$, XUEDONG HOU ${ }^{2}$, GONGBI FU $^{2}$, NING AN ${ }^{2}$ and LIPING WANG ${ }^{1}$ \\ ${ }^{1}$ Department of Otorhinolaryngology, Shengjing Hospital, China Medical University, Shenyang, Liaoning 110004; \\ ${ }^{2}$ Department of Otorhinolaryngology, Fengtian Hospital, Shenyang Medical University, Shenyang, Liaoning 110024; \\ ${ }^{3}$ Department of Pancreatic Surgery, First Hospital of China Medical University, Shenyang, Liaoning 110001, P.R. China
}

Received May 22, 2015; Accepted April 4, 2016

DOI: $10.3892 / \mathrm{mmr} .2016 .5204$

\begin{abstract}
Genistein and trichostatin A (TSA) are two chemotherapeutic compounds with antitumor effects in different types of cancer cell. However, the effects of genistein and TSA on the HEp-2 laryngeal cancer cell line remain to be fully elucidated. In the present study, it was found that genistein and TSA inhibited cell growth and cell migration, and promoted apoptosis in the HEp-2 laryngeal cancer cell line. The HEp-2 cells were treated with genistein, TSA or the two compounds in combination. Cell proliferation and apoptosis were measured using an MTT assay, Annexin V/propidium iodide staining and a TUNEL assay. Cell invasion was determined using a Matrigel-based Transwell assay. Western blotting was used to examine the activation of the Akt pathway and the expression levels of pro-or anti-apoptotic proteins. Treatment with either genistein or TSA alone mildly inhibited cell viability, growth and invasion, and induced the apoptosis of the laryngeal cancer cells, whereas more marked effects were observed in the cells treated with the combination of the two compounds. In addition, genistein reversed endothelial growth factor-induced epithelial-mesenchymal transition (EMT) in the HEp-2 cells, the effect of which were was further increased by joint application with TSA. Treatment of the HEp-2 cells with genistein and TSA led to a significant reduction in the phosphorylation of Akt and activation of its downstream target, and resulted in peroxisome proliferator-activated receptor- $\gamma$ cleavage, increased expression of $\mathrm{B}$ cell lymphoma-2 (Bcl-2)-associated $\mathrm{X}$ protein and reduced the expression of $\mathrm{Bcl}-2$. In conclusion, the present study demonstrated that, with the involvement of TSA, genistein exhibited substantial advantages in inhibiting laryngeal carcinoma cell growth, invasion and EMT, and
\end{abstract}

Correspondence to: Dr Liping Wang, Department of Otorhinolaryngology, Shengjing Hospital, China Medical University, 36 Sanhao Street, Heping, Shenyang, Liaoning 110004, P.R. China E-mail: lipingwang15@163.com

Key words: genistein, trichostatin A, laryngeal carcinoma, epithelial-to-mesenchymal, apoptosis, HEp-2 induced apoptosis, compared with genistein treatment alone, which occurred through the regulation of Akt activation and the apoptotic pathway.

\section{Introduction}

Laryngeal carcinoma is the second most common neoplasm occurring in the head and neck region, with high levels of invasion and metastasis $(1,2)$. Due to low survival rates, determining how to improve quality of life and survival rates in patients with laryngeal cancer remains a significant challenge (3). The most frequently used treatment is chemotherapy, and chemotherapeutic agents, including cisplatin, gefitinib, taxol and doxorubicin, are used experimentally or clinically to improve the prognosis of patients with laryngeal cancer $(4,5)$. However, due to multidrug resistance-induced therapeutic failure and poor prognosis, more efficient chemotherapeutic agents and novel agent combinations are required to improve the treatment of laryngeal cancer.

Genistein, a soy-derived isoflavone, exhibits broad tumor suppressive effects in various types of human cancer by regulating several signaling pathways, including the mitogen-actived protein kinase, Akt and Janus kinase/signal transducers and activators of transcription (6-8). However, current knowledge of its role in regulating laryngeal cancer cell biofunction is limited to its inhibition of HEp-2 cell growth (9), and more detailed investigations of its function and mechanism are required.

As the combining of chemotherapeutic agents has been a common and effective clinical strategy for cancer therapy, the present study aimed to identify compounds, which can improve the effects of genistein in preventing the progression of laryngeal cancer. Epigenetic modifications have been found to be important in cancer development and, correspondingly, compounds regulating this event are currently considered as promising anticancer agents $(10,11)$. Trichostatin A (TSA) is known to be a specific inhibitor of histone deacetylases, and effectively arrests cell growth and induces apoptosis in various types of human cancer cell, including prostate, colorectal, breast and gastric cancer $(12,13)$. At present, there is a lack of information regarding the effects of TSA on laryngeal carcinoma, therefore, the present study aimed to investigate the combined effects of genistein and TSA on laryngeal cancer treatment. 
The results of the present study, demonstrated that TSA improved genistein-induced tumor suppression in human laryngeal cancer cells. The data obtained confirmed that TSA potentiated the anti-laryngeal cancer action of genistein via promoting apoptosis and reversing epithelial-mesenchymal transition (EMT).

\section{Materials and methods}

Antibodies and growth factors. The antibodies used in the present study included monoclonal rabbit anti-E-cadherin (cat. no. 24E10), monoclonal rabbit anti-vimentin (cat. no. 5741S), monoclonal rabbit anti-B cell lymphoma (Bcl)-2 (cat. no. 2870) and monoclonal rabbit anti-Bcl-2-associated X protein (Bax; cat. no. 14796) primary antibodies obtained from Santa Cruz Biotechnology, Inc. (Dallas, TX, USA). Monoclonal mouse anti-PARP (1:1,000; cat. no sc-8007; Santa Cruz Biotechnology, Inc.), monoclonal rabbit Akt (1:1,000; cat. no. 4691; Cell Signaling Technology, Inc., Danvers, MA, USA), monoclonal rabbit anti-pAkt (1:500; cat. no. 4060; Cell Signaling Technology, Inc.), monoclonal mouse anti-S6 (1:1,000; cat. no. 2317; Cell Signaling Technology, Inc.), monoclonal rabbit anti-pS6 (1:200; cat. no. 4858; Cell Signaling Technology, Inc.), monoclonal rabbit anti-4eBP (1:100; cat. no. 9452; Cell Signaling Technology, Inc.), monoclonal rabbit anti-p4eBP (1:100; cat. no. 2855; Cell Signaling Technology, Inc.) and monclonal rabbit anti-GAPDH (1:2,000; cat. no. sc-25778; Santa Cruz Biotechnology, Inc.) primary antibodies were also used. Polyclonal goat anti-rabbit (cat. no. A21020) and goat anti-mouse (cat. no. A21010) secondary antibodies were purchased from Abbkine, Inc. (Redlands, CA, USA). Transforming growth factor (TGF)- $\beta 1$ and epidermal growth factor (EGF) were purchased from PeproTech, Inc. (Rocky Hill, NJ, USA). Genistein and TSA were obtained from Sigma-Aldrich (St. Louis, MO, USA) and were dissolved in dimethyl sulfoxide (DMSO; Sangon Biotech Co., Ltd., Shanghai, China).

Cell culture. The HEp2 human laryngeal cancer cell line was purchased from SIBS Cell Bank (Shanghai, China) and cultured in high-glucose Dulbecco's modified Eagle's medium (DMEM; Gibco; Thermo Fisher Scientific, Inc., Waltham, MA, USA) containing $10 \%$ fetal bovine serum (FBS; Gibco; Thermo Fisher Scientific, Inc.). The medium was renewed every day and the cells were passaged prior to reaching confluence.

EMT induction in the HEp-2 cells. EMT was achieved when the $1 \times 10^{4} \mathrm{HEp}-2$ cells were stimulated with EGF $(30 \mathrm{ng} / \mathrm{ml})$ for $48 \mathrm{~h}$ at $37^{\circ} \mathrm{C}$. Subsequently, the cells were treated with genistein $(20 \mu \mathrm{M})$ or genistein+TSA $(20 \mu \mathrm{M}$ : $100 \mathrm{nM})$, in the presence or absence of EGF, for another $48 \mathrm{~h}$ at $37^{\circ} \mathrm{C}$. Alterations in cell morphology were documented by visualization using an inverted phase contrast microscope (DFC500; Leica Microsystems GmbH, Wetzlar, Germany).

Western blotting. The HEp-2 cells were harvested and washed once with phosphate-buffered saline (PBS), and further lysed in radioimmunoprecipitation assay buffer with phenylmethylsulfonyl fluoride (Sangon Biotech Co., Ltd.). Protein concentrations were determined using a Pierce BCA protein assay kit (Thermo Fisher Scientific, Inc.). SDS-PAGE (12\% gel) was utilized to separate equal quantities $(20 \mu \mathrm{g})$ of the proteins, which were then electrically transferred onto nitrocellulose membrane (GE Healthcare Life Sciences, Chalfont, UK), and blocked with 5\% fat free milk in Tris-buffered saline with $0.05 \%$ Tween (TBST) buffer for $1 \mathrm{~h}$ at room temperature. Individual membranes were further incubated with appropriately diluted primary antibodies $(1: 1,000)$ overnight at $4^{\circ} \mathrm{C}$. Horseradish peroxidase (HRP)-conjugated secondary antibodies $(1: 5,000)$ were then applied for $2 \mathrm{~h}$ at room temperature following three intensive washes in TBST. The HRP substrate gives rise to the final images using electrochemiluminescence (Western Bright kit; cat. no. R-03031-D25; Advansta, Inc., Menlo Park, CA, USA), visualized following X-film development [Fujifilm (China) Investment Co., Ltd., Shanghai, China].

Invasion assay. Transwell inserts (Corning Incorporated, Corning, NY, USA) were pre-coated with $50 \mu$ l Matrigel (BD Biosciences, San Jose, CA, USA) and placed into wells of a 12-well culture plate. Equal quantities of HEp-2 cells $\left(2 \times 10^{5}\right)$ were seeded into the upper chamber in culture medium, and cell invasion was determined at $24 \mathrm{~h}$ following the addition of the chemo-attractant, of FBS, to the lower chamber. The invasive cells were visualized using $0.1 \%$ crystal violet staining (Sangon Biotech Co., Ltd.) following fixation with $4 \%$ paraformaldehyde (PFA) and imaged using a DFC500 microscope (Leica Microsystems GmbH). Subsequent quantification was performed using the mean numbers of cells counted from five randomly selected fields (magnification, x200). The data were summarized from three independent experiments.

Wound healing assay. HEp-2 cells $\left(2 \times 10^{5}\right)$ were seeded in 6 -well plates. After $24 \mathrm{~h}$, the cells were serum starved for $12 \mathrm{~h}$. A linear wound was created using a pipette tip and the cells were washed three times using PBS. Subsequently, the cells were cultured in medium supplemented with $1 \%$ FBS. Wounds were then assessed after $24 \mathrm{~h}$. Images of 3 random fields were captured $24 \mathrm{~h}$ after the scratch wound using a Leica DFC500 microscope (magnification, x200). The migration distance (arbitrary units) was determined as reduction in the wounds gap using NIH Image J software (version 1.32; National Institute of Health, Bethesda MD, USA).

Cell viability. Equal quantities of HEp-2 cells $\left(2.0 \times 10^{4}\right)$ were seeded into wells of a 96-well plate and further cultured for $24 \mathrm{~h}$. The cells were then treated with genistein $(1,10,20$, 50 and $100 \mu \mathrm{M})$ or TSA $(10,50,100,200$ and $500 \mathrm{nM})$ for another $48 \mathrm{~h}$ at $37^{\circ} \mathrm{C}$. This was followed by incubation for $4 \mathrm{~h}$ with MTT (Sangon Biotech Co., Ltd.) at a final concentration of $0.5 \mathrm{mg} / \mathrm{ml}$, following which $100 \mu \mathrm{l}$ DMSO was added following removal of the medium. Cell viability was determined by measuring optical density (OD) at $490 \mathrm{~nm}$ (Bio-Rad Laboratories, Inc., Hercules, CA, USA). At least three independent experiments were performed, and the final inhibitory rates were determined according to the following formula: Inhibitory rate $(\%)=\left[1-\left(\mathrm{OD} 490_{\text {compound }} / \mathrm{OD} 490_{\text {solvent }}\right)\right] \mathrm{x} 100 \%$.

TUNEL assay. The HEp-2 cells were allowed to grow to $80 \%$ confluence and fixed with $4 \%$ PFA for $30 \mathrm{~min}$, followed 
by permeation with $0.1 \%$ Triton X-100 (Sangon Biotech Co., Ltd.)/PBS on ice for $2 \mathrm{~min}$. Following three washes with PBS, TUNEL reagent was applied for the detection of apoptotic events, according to manufacturer's protocol (Beyotian Biotechnology Co., Ltd., Shanghai,. China) and 4',6-diamidino-2-phenylindole (Sangon Biotech Co., Ltd.) was used to stain the nuclei. The staining results were visualized and documented using an Olympus DP71X immunofluorescent microscope (Olympus Corporation, Tokyo, Japan).

Annexin V-fluorescein isothiocyanate (FITC) staining and fluorescence-activated cell sorting (FACS). The staining protocol was performed, according to the manufacturer's protocol (BD Biosciences). Briefly, $5 \times 10^{5}$ cells were harvested centrifugation at $1,000 \mathrm{x}$ g for $5 \mathrm{~min}$ at room temperature and suspended in $195 \mu \mathrm{l}$ binding buffer, followed by incubation for 10 min with $5 \mu \mathrm{l}$ Annexin V-FITC at room temperature in the dark. Following additional centrifugation at $1,000 \mathrm{x} \mathrm{g}$ for $5 \mathrm{~min}$ at room temperature, the cells were resuspended in $190 \mu \mathrm{l}$ binding buffer with $10 \mu \mathrm{l}$ propidium iodide (Sangon Biotech Co., Ltd.) under gentle agitation. FACS was performed using the BD Accuri C6 (BD Biosciences) and FlowJo software (version 7.6.2; Tree Star, Inc., Ashland, OR, USA) to detect apoptotic events.

$E d U$ assay. The EdU incorporation assay was performed using an EdU assay kit (Guangzhou RiboBio Co., Ltd., Guangzhou, China) according to the manufacturer's instructions. Briefly, HEp-2 cells $\left(2 \times 10^{5}\right)$ were incubated with DMEM containing $50 \mathrm{mM}$ EdU for $2 \mathrm{~h}$. The nuclei were stained with Hoechst 33342 (5 $\mu \mathrm{g} / \mathrm{ml}$; Sigma-Aldrich) and the images were acquired with an Olympus DP71X microscope.

Statistical analysis. SPSS software (version 16; SPSS, Inc., Chicago, IL, USA) was used to perform statistical analysis. Data are expressed as the mean \pm standard error of the mean, and one-way analysis of variance was used. $\mathrm{P}<0.05$ was considered to indicate a statistically significant difference.

\section{Results}

TSA enhances the inhibitory effect of genistein on HEp-2 cell growth. To evaluate whether genistein or TSA affect cell viability, the HEp-2 cells were separately incubated with the compounds, at a range of concentrations, for 2 days. Using an MTT assay, the half maximal inhibitory concentration $\left(\mathrm{IC}_{50}\right)$ of genistein was determined as $\sim 20 \mu \mathrm{M}$, and TSA as $100 \mathrm{nM}$ (Fig. 1A). The combined treatment strategy of genistein $(20 \mu \mathrm{M})$ and TSA $(100 \mathrm{nM})$ led to a more marked reduction in HEp-2 cell viability, compared with the individual compound treatments. This occurred in a time-dependent manner between 12 and 48 h (Fig. 1B-D). To confirm this, a 5-ethynyl-20-deoxyuridine incorporation assay was performed. Cell proliferation was detected following treatment with genistein and TSA, alone or in combination, for $24 \mathrm{~h}$. It was found that the combined treatment with genistein $(20 \mu \mathrm{M})$ and TSA $(100 \mathrm{nM})$ resulted in a marked decrease in cell proliferation (Fig. 1E and F). A similar result was obtained in the single colony formation assay, indicating that the combination treatment had a more marked effect in impairing the ability of HEp-2 cells to form colonies, compared with any of the individual treatments. (Fig. $1 \mathrm{G}$ and $\mathrm{H}$ ).

TSA has an additive effect on the antagonistic effects of genistein on EGF-induced EMT in HEp-2 cells. To assess the role of genistein $(20 \mu \mathrm{M})$, TSA $(100 \mathrm{nM})$ and genistein+TSA (20 $\mu \mathrm{M}: 100 \mathrm{nM}$ ) on EGF-induced EMT phenotype and mensenchymal gene expression levels, the HEp-2 cells were treated with genistein $(20 \mu \mathrm{M})$, TSA $(100 \mathrm{nM})$ or geinstein+TSA $(20 \mu \mathrm{M}: 100 \mathrm{nM})$ in the presence or absence of EGF for $48 \mathrm{~h}$. EGF increased the numbers of cells with spindle-like shapes and loss of cell-cell contact, a typical phenotype of EMT (Fig. 2A; red arrow), associated with an increase in the expression of vimentin (Fig. 2B). Treatment with genistein alone led to EGF-induced EMT (Fig. 2A; apoptotic cells indicated by green arrow), increased the expression of E-cadherin and reduced the expression of vimentin (Fig. 2B), these effects were amplified by treatment with genistein+TSA. In addition, the results of the Transwell invasion assay revealed that genistein inhibited EGF-induced HEp-2 cell invasion, although this was less marked, compared with combined treatment with genistein and TSA (Fig. 2C). Subsequently, a scratch wound migration assay was performed. A marked decrease in cell migration was observed $48 \mathrm{~h}$ following treatment with genistein+TSA (Fig. 2D).

TSA promotes genistein-induced HEp2 cell apoptosis. Following the observation of visible apoptotic cellular morphology (Fig. 3A) and peroxisome proliferator-activated receptor $\gamma$ activation in the genistein+TSA treated HEp2 cells (Fig. 3B), the present study investigated the extent to which genistein and TSA induce HEp2 cells apoptosis. Following $48 \mathrm{~h}$ treatment with genstein, TSA or geinstein+TSA, Annexin V/PI staining demonstrated an elevation in the apoptotic population of cells in the genistein- and the TSA-treated cells (14.5 and $11.2 \%$, respectively), however, a more marked increase of $40.3 \%$ was detected in the cells in the combined treatment group (Fig. 3C and D). In addition, the TUNEL assay revealed similar results to those observed in the Annexin V/PI staining, with the most marked levels of apoptosis observed in the combined treatment group (Fig. 3E and F).

Combined treatment of TSA and genistein inhibits Akt signaling in HEp2 cells. As the Akt pathway is central in controlling cell apoptosis, the present study further detected the effects of genistein, TSA and genistein+TSA on the activation of Akt and its downstream molecules. Although the phosphorylation levels of Akt, S6 and 4eBP1 were detectable in the genistein- and TSA-treated cells, inhibition appeared more marked following combined treatment with Genstein and TSA (Fig. 4A), leading to an additive increase in the protein expression of pro-apoptotic Bax and decrease in the protein expression of anti-apoptotic Bcl-2 (Fig. 4B).

\section{Discussion}

Although currently used chemotherapeutic treatments improve the prognosis of patients, the identification of improved 
A

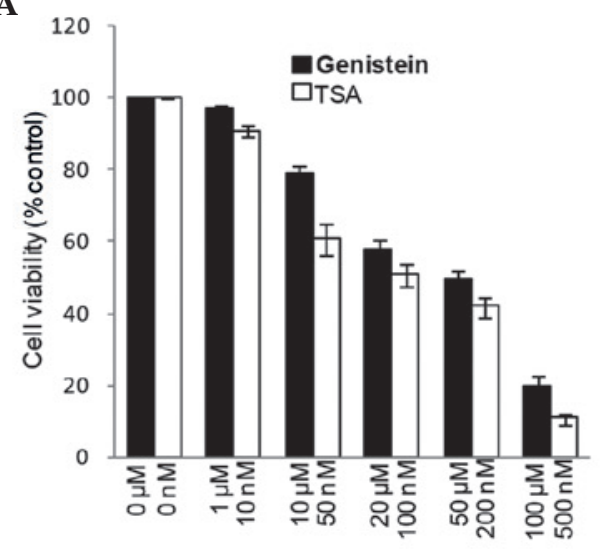

D

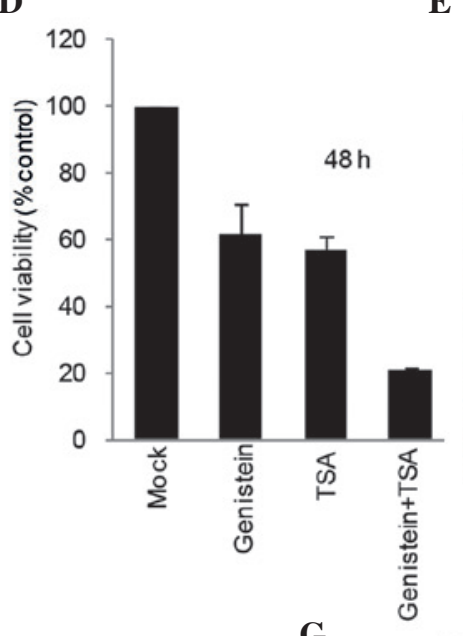

G

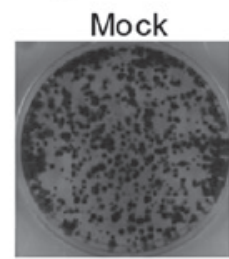

TSA

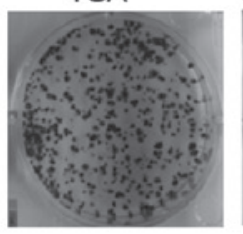

B

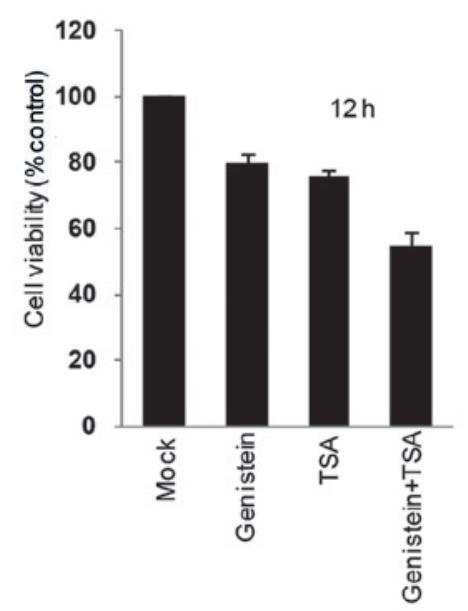

C

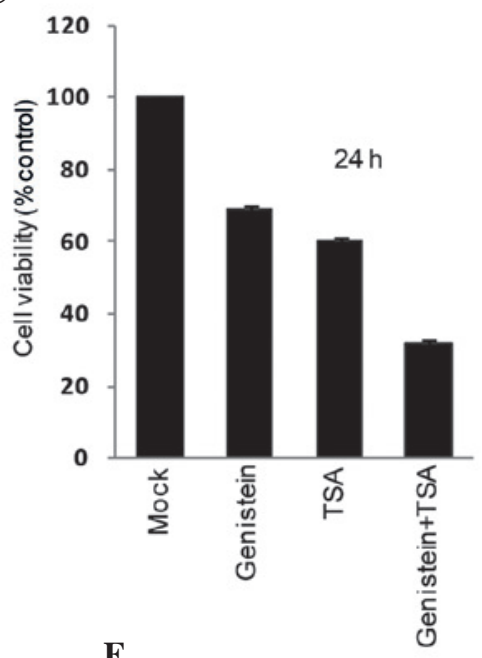

F

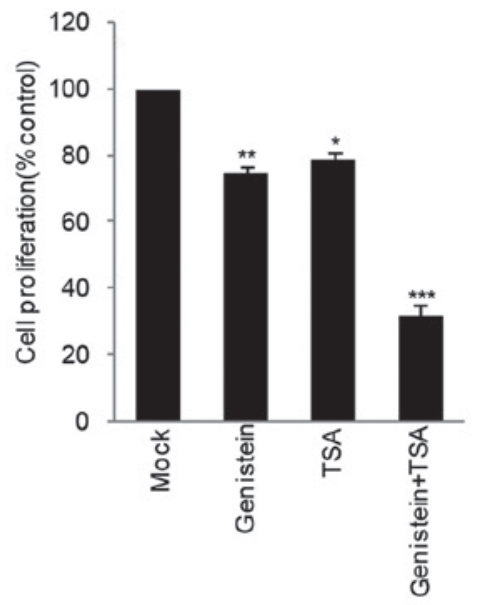

Figure 1. TSA has a synergistic inhibitory effect on genistein-induced HEp-2 cell growth impairment. An MTT assay was used to detect the effects of genistein and TSA on HEp-2 cell viability (A) individually and (B-F) in combination, to determine their synergistic effects at indicated measuring time points. The HEp-2 cells treated with genistein and TSA alone and in combination were stained with EdU. The nuclei were also visualized using Hoechst 33342 staining. The percentage of cell proliferation is expressed as the ratio of EdU-positive cells to total Hoechst 33342-positive cells. The number of untreated cells was set as $100 \%$. Magnification, x200. (G) Representative images showing the effects of TSA, genistein and TSA+genistein on HEp-2 single cell colony formation, with $(\mathrm{H})$ quantification presented. All data were obtained from at least three independent experiments and are presented as the mean \pm standard error of the mean. ${ }^{*} \mathrm{P}<0.05,{ }^{* *} \mathrm{P}<0.01$ and ${ }^{* * *} \mathrm{P}<0.001$, vs. Mock. TSA, trichostatin A.

combined treatment strategies remains a requirement for improving the survival rates of patients diagnosed with laryngeal cancer. The present study aimed to identify efficient drugs for use in laryngeal cancer therapy, and to evaluate whether or not the combined effects of these drugs offer a novel therapeutic strategy for the treatment of laryngeal cancer.

As common chemotherapeutic drugs, genistein and TSA separately exert tumor suppressive effects in different 
A
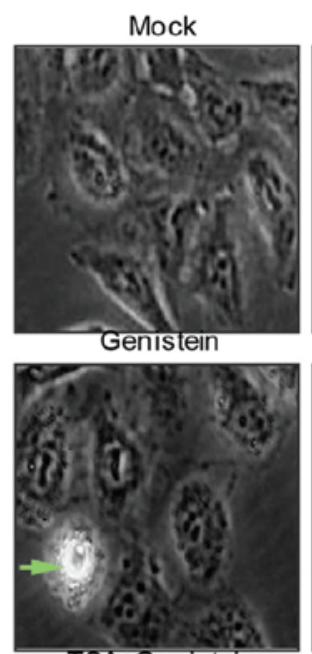

TSA+Genistein

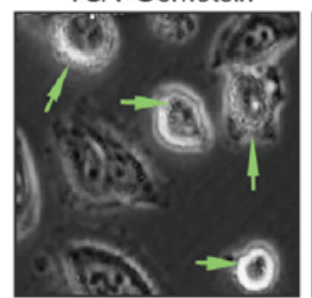

C

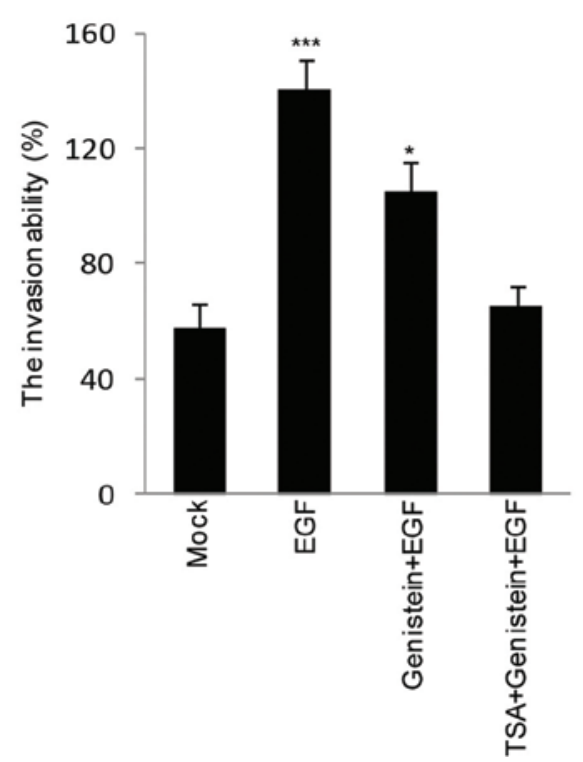

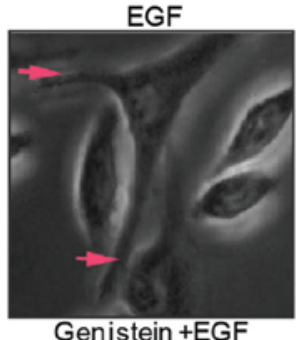
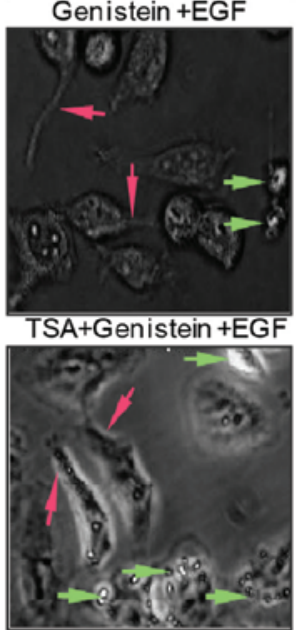

D
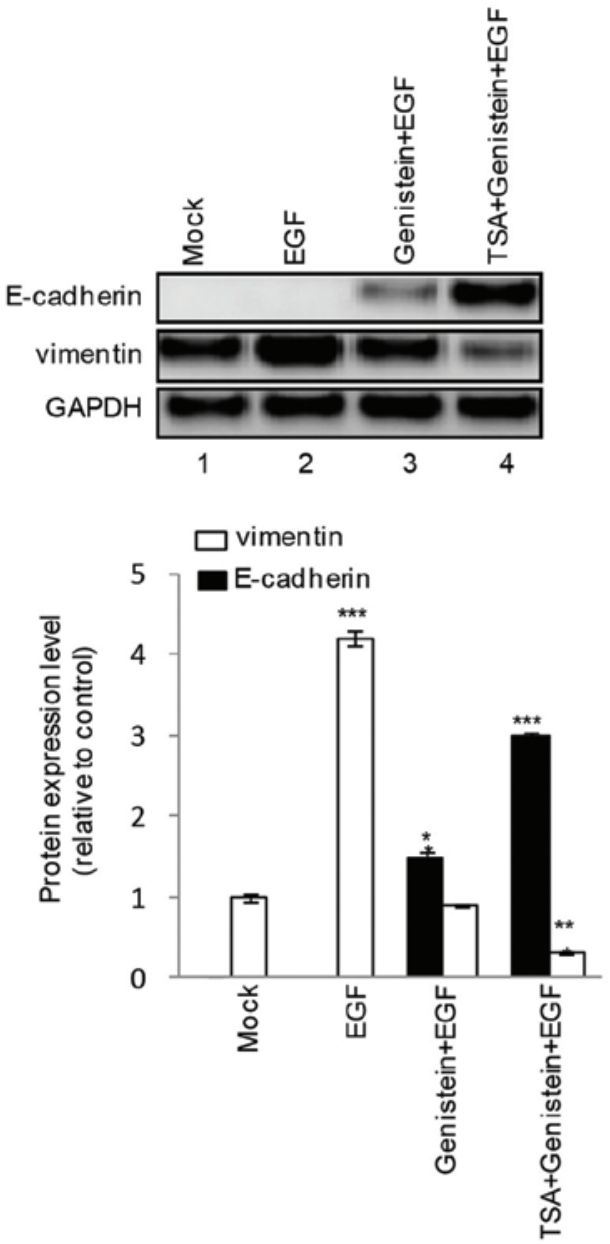

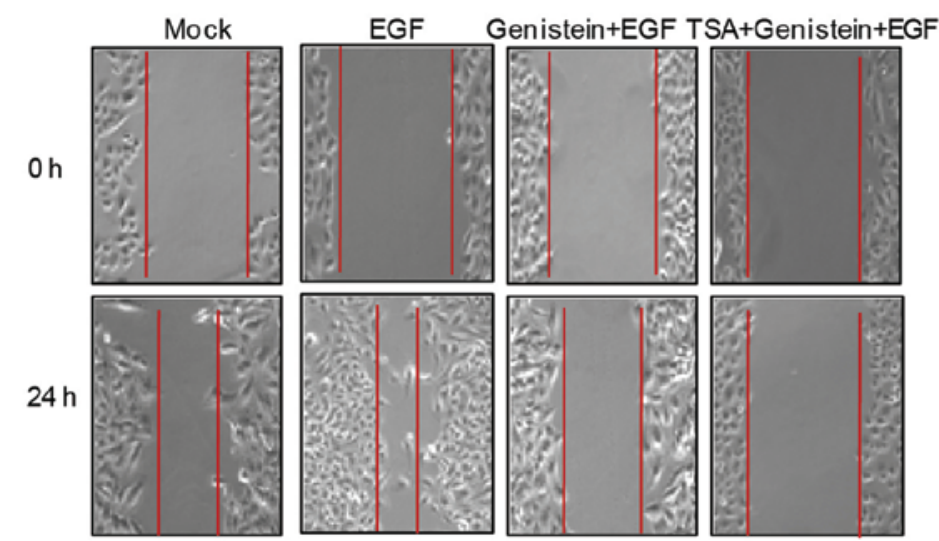

Figure 2. Combined TSA and genistein treatment prevents EGF-induced HEp-2 cell epithelial-mesenchymal transition and invasion. HEp-2 cells were treated with EGF in the presence or absence of TSA, genistein or TSA+genistein for $48 \mathrm{~h}$, and (A) corresponding cell morphological changes were recorded. Green arrows indicate apoptotic cells and red arrows indicate migrating cells. Magnification, x400. (B) Expression levels of E-caderin and vimentin were detected using western blotting. (C) Cell invasive ability was examined using a Transwell-based invasion assay. (D) HEp-2 cells were treated with EGF in the presence of absence of TSA, genistein or TSA+genistein for $24 \mathrm{~h}$, and a scratch wound migration assay was performed. The data are summarized from three independent experiments and are expressed as the mean \pm standard error of the mean. ${ }^{*} \mathrm{P}<0.05,{ }^{* *} \mathrm{P}<0.01$ and ${ }^{* * *} \mathrm{P}<0.001$, vs. Mock. TSA, trichostatin $\mathrm{A}$; EGF, epidermal growth factor.

types of human cancer, including breast (8), gastric (13), lung $(14,15)$ and prostate cancer $(16)$, through various mechanisms. However, the combined consequence of the two compounds, and their respective effects on the biological function of laryngeal cancer cells remain to be fully elucidated. In the present study, genistein and TSA were 
$\mathbf{A}$

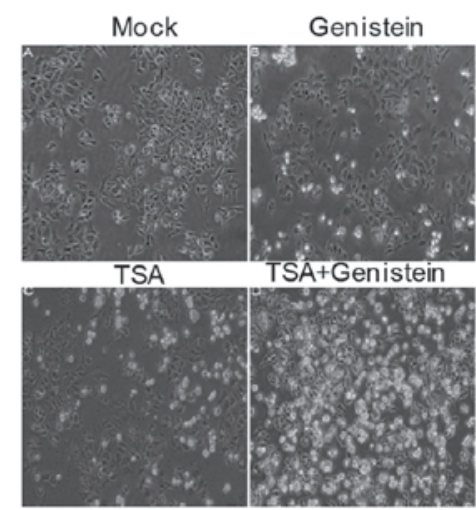

C
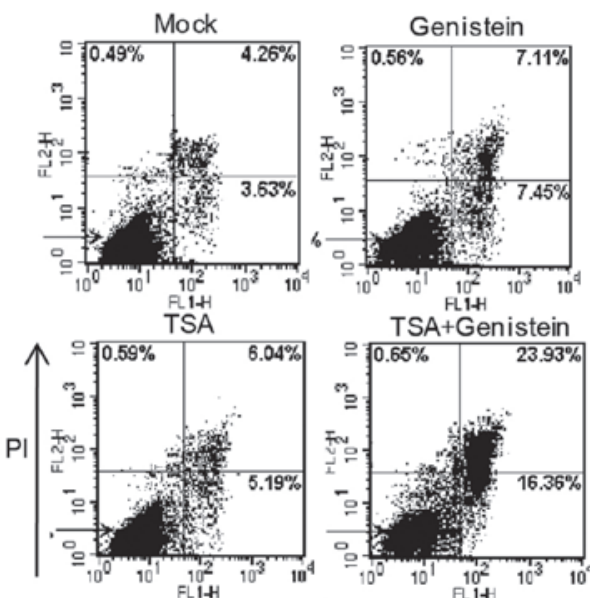

TSA+Genistein

$\mathbf{E}$
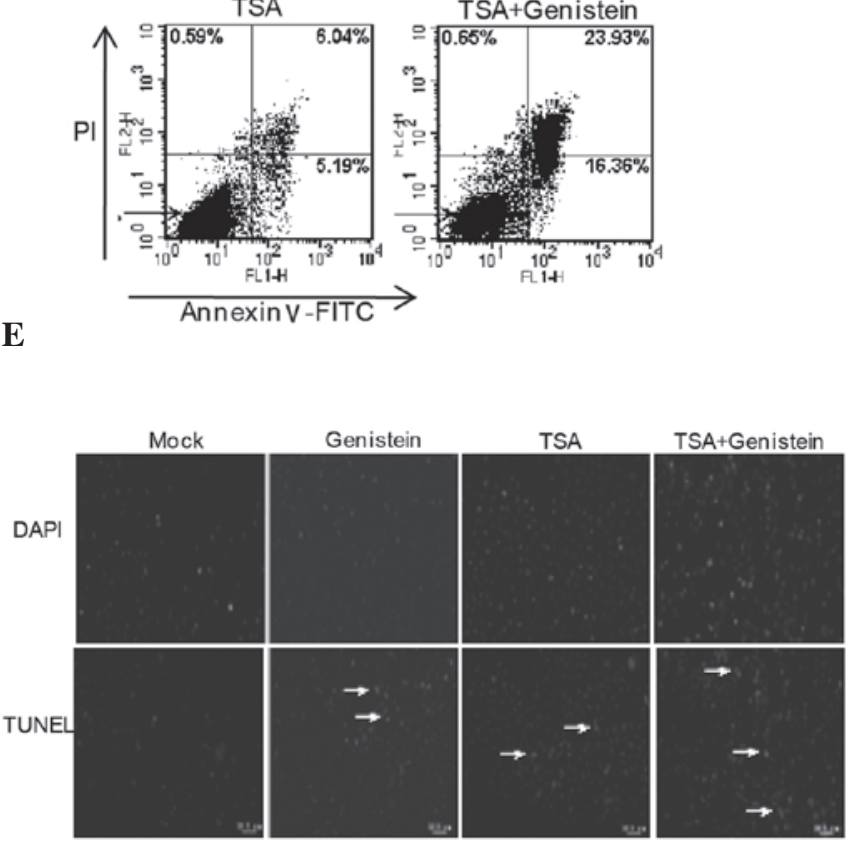

B
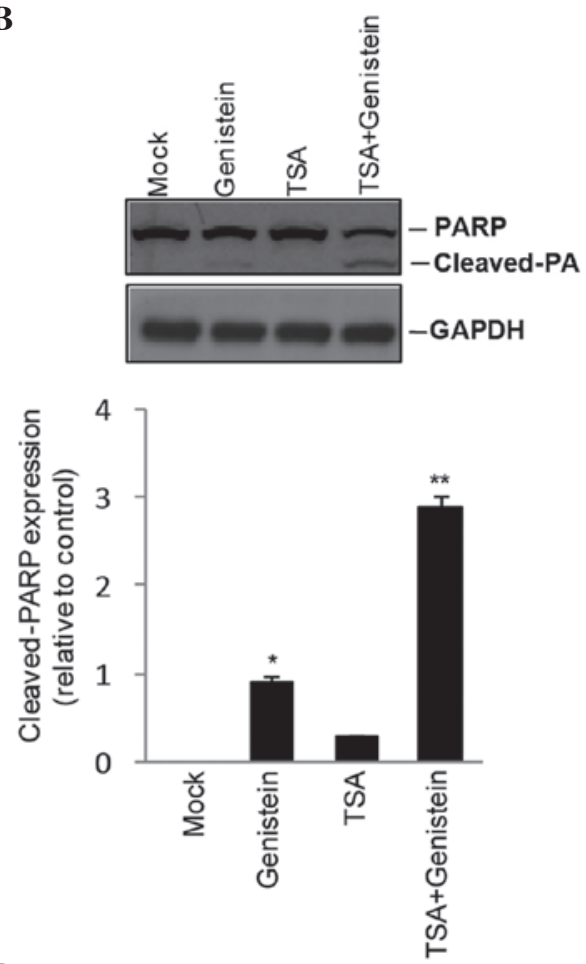

D

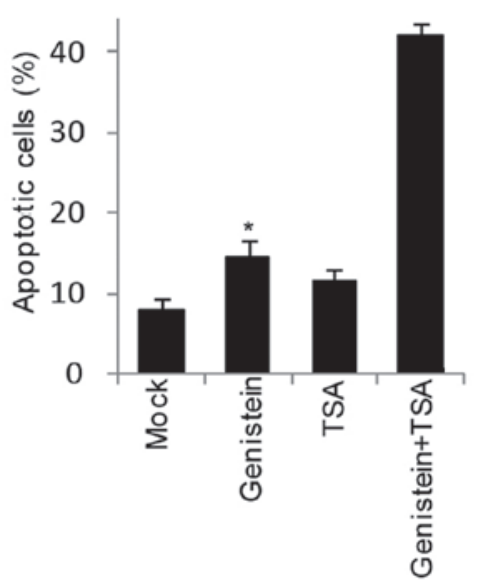

F

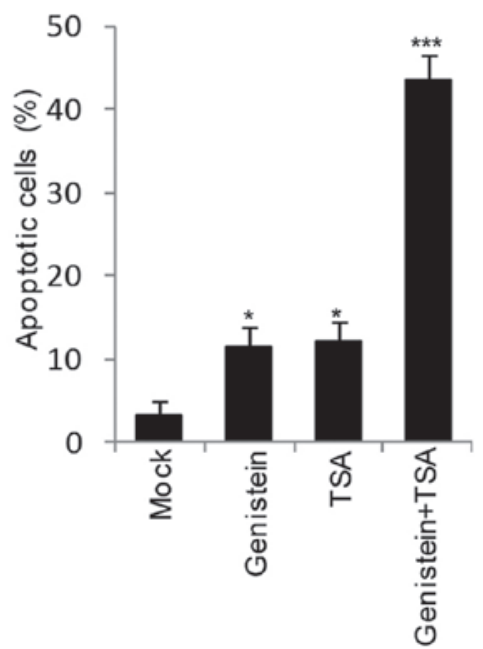

Figure 3. Effects of combined TSA and genistein treatment on HEp-2 cells apoptosis. Following treatment for $48 \mathrm{~h}$ with TSA, genistein or TSA+genistein, the HEp-2 cells exhibited a (A) pro-apoptotic phenotype (magnification, x200) with (B) PPAR $\gamma$ activation, detected using western blotting. Fluorescence-activated cell sorting analysis with (C and D) PI and Annexin VI staining and a (E and F) TUNEL assay (magnification, x200) were utilized to examine the combined effects of TSA+genistein on HEp-2 apoptosis. The data were obtained from three independent experiments and expressed as the mean \pm standard error of the mean. ${ }^{*} \mathrm{P}<0.05,{ }^{* * *} \mathrm{P}<0.01$ and ${ }^{* * *} \mathrm{P}<0.001$, vs. Mock. TSA, trichostatin A; PPAR $\gamma$, peroxisome proliferator-activated receptor $\gamma$; PI, propidium iodide; FITC, fluorescein isothiocyanate. 
A
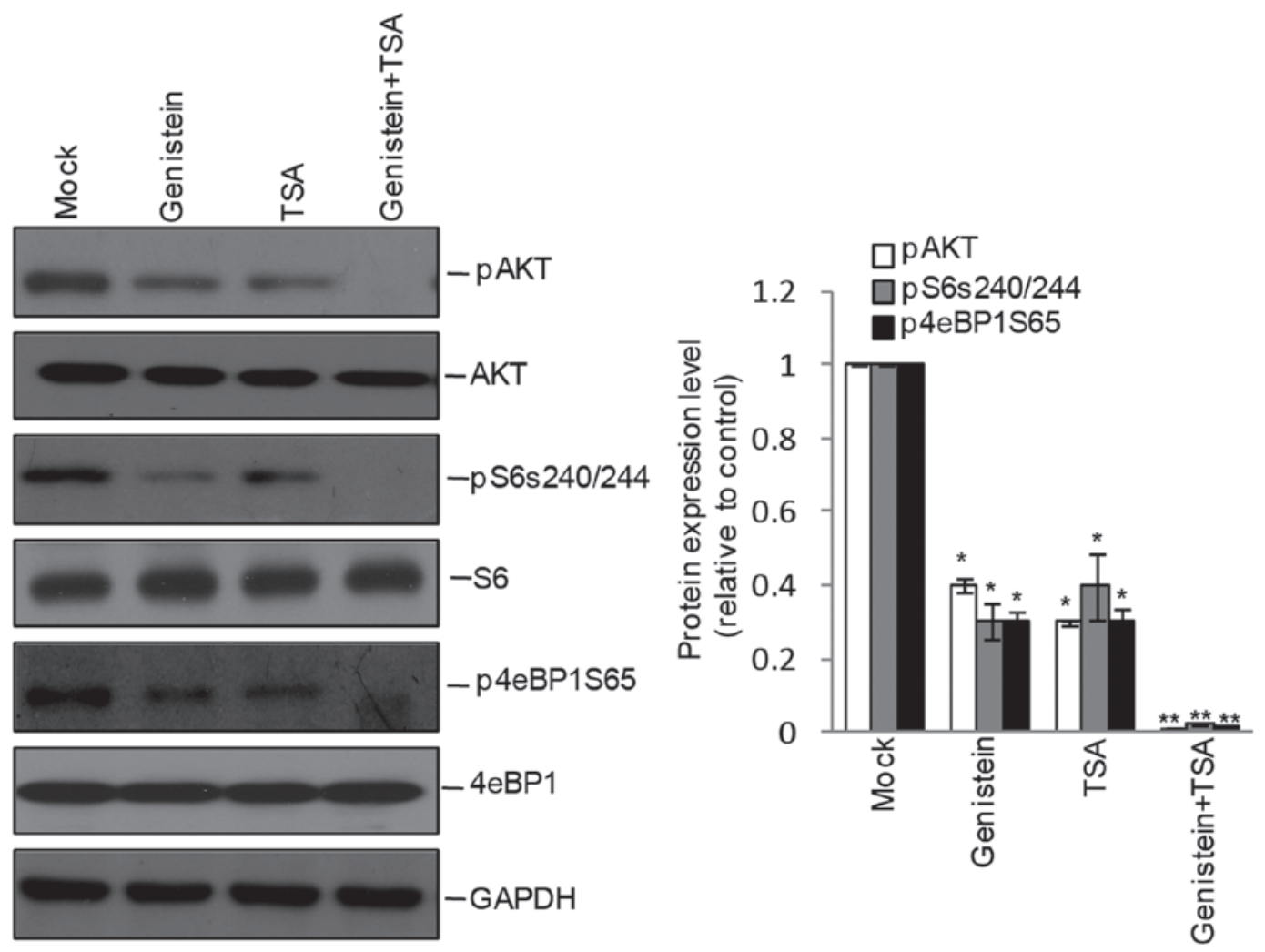

\section{B}
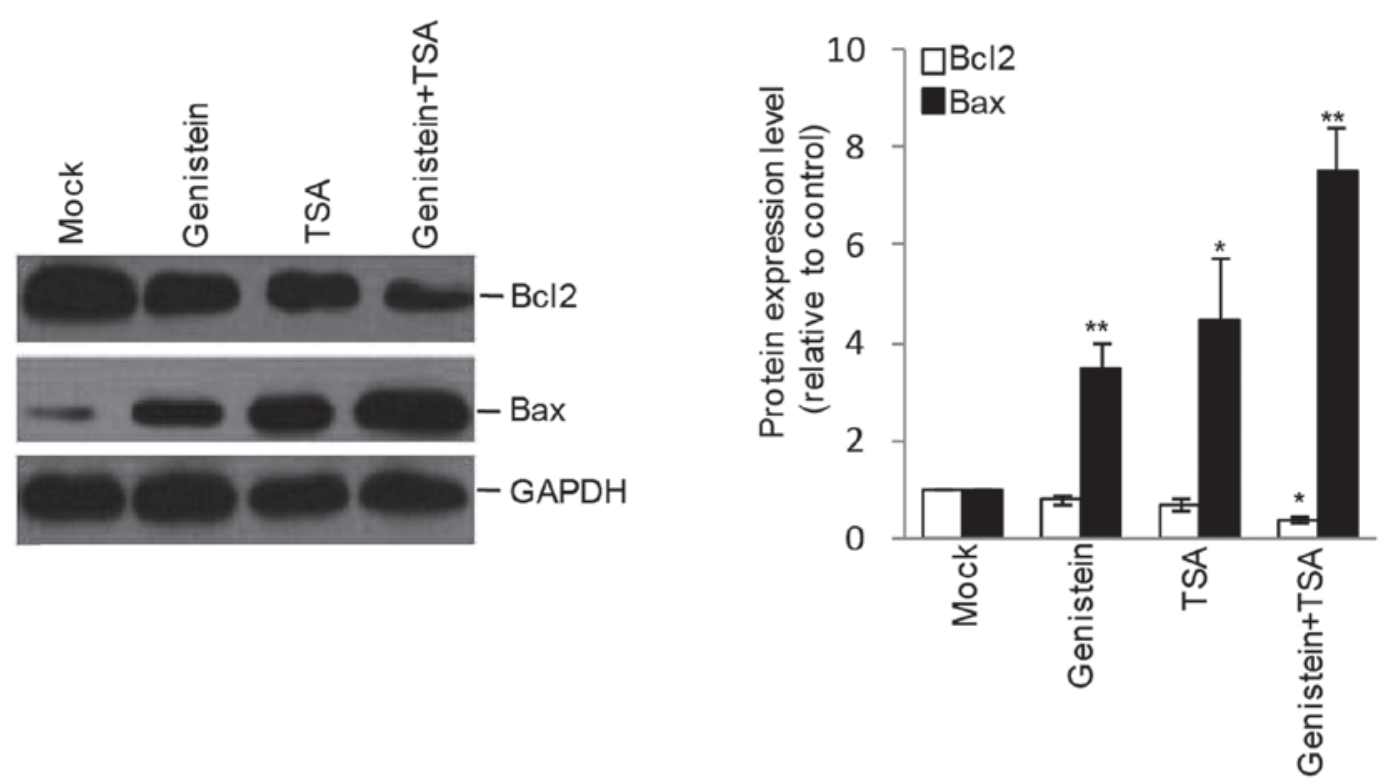

Figure 4. Effects of combined treatment of TSA and genistein on Akt signaling. The HEp-2 cells were incubated with TSA, genistein or TSA+genistein for $48 \mathrm{~h}$, and western blotting was used to examine the (A) activity of the Akt pathway by the levels of phosphorylated (p) Akt, S6 and 4eBP1. (B) Apoptotic-associated protein expression of $\mathrm{Bcl}-2$ and Bax. Quantitative measurements were obtained from 3 independent experiments and are presented as the mean \pm the standard error of the mean. ${ }^{*} \mathrm{P}<0.05,{ }^{* * *} \mathrm{P}<0.01$ and ${ }^{* * *} \mathrm{P}<0.001$ vs. Mock. TSA, TSA, trichostatin A; Bcl-2, B cell lymphoma-2; Bax, Bcl-2-associated X protein.

found to have significant, although mild effects leading to reduced cell viability, colony formation and invasion, and increased apoptosis. Compared with other cancer cells, the tumor-repressing effects of Genstein and TSA on the HEp2 cells differed, which may explained by the varied genetic backgrounds of cells. The present study also investigated the combined contribution of genistein and TSA on laryngeal cancer growth. The concentrations of genistein and TSA used were 20 and $100 \mathrm{nM}$, respectively, determined as the $\mathrm{IC}_{50}$ values for the individual compounds in the cell viability assay, to generate this level of inhibition without any further additive cell toxicity and avoid false positive effects caused by additive cell toxicity. Compared with the effects of the individual drugs, the combined treatment led to marked 
attenuation in HEp-2 cell viability, colony formation and invasion, and marked promotion of apoptosis, indicating an efficient strategy for suppressing HEp-2 cell growth.

The present study then aimed to examine the mechanism underlying genistein+TSA-induced suppression of laryngeal cancer cells. Due to the marked reduction in invasive ability observed in the HEp2 cells following genistein+TSA treatment, it was hypothesized that this may link to HEp2 cell EMT, a crucial process with a fundamental role in controlling cell migration, invasion and metastasis (17). In the present study, EGF was used to trigger HEp2 cell EMT, which led to increased cell invasion accompanied by an upregulation in the mensenchymal maker gene, vimentin. Furthermore, the data experimentally confirmed that genistein alone was able to partially antagonize the EGF-induced cell invasion and expression of vimentin and induce the expression of the epithelial gene, E-cadherin, in the laryngeal cancer HEp-2 cells. These effects have also been observed in human hepatocellular carcinoma cells (18) and prostate cancer cells (16). In addition, combined treatment with TSA, a compound previously showing EMT suppression in prostate cancer PC3 cells (17) and breast and gastric cancer cells, genistein completely reversed EGF-induced EMT, indicating the possibility that genistein+TSA suppressed cell growth and invasion through regulating cellular EMT. In addition, although the induction of apoptosis by genistein or TSA has been described in previous reports for other cancer cells, their effects on laryngeal cancer cell apoptosis remain to be elucidated. In the present study, it was observed that genistein or TSA had weak effect on the promotion of cell apoptosis, however, when the two compounds were applied in combination, a marked, but not additive, acceleration in apoptotic rate was observed in the HEp2 cells. In view of the signaling pathway, the present study confirmed that genistein or TSA partially suppressed the activation of Akt and its downstream molecules, however, their activation was completely eliminated by the combined treatment of genistein with TSA. The increased protein expression of pro-apoptotic Bax and a decreased protein expression of anti-apoptotic Bcl-2 were also observed. Therefore, genistein and TSA jointly promoted laryngeal cancer cell apoptosis via silencing the activation of the Akt pathway.

Although the present study demonstrated that the combination of genistein+TSA exerted anti-laryngeal tumor effects in cultured HEp-2 cells, fundamental in vivo investigations are required. For this purpose, future in vivo experiments aim to evaluate the pharmacological efficiency of genistein+TSA on laryngeal cancer therapy in experimental animals.

In conclusion, the present study was the first, to the best of our knowledge, to report that genistein and TSA possess anti-aryngeal cancer properties, and further identified the novel drug combination of genistein+TSA for laryngeal cancer therapy, which demonstrated marked advantages in suppressing cell growth and invasion, and promoting apoptosis via the reversal of EMT and quenching of Akt activation in HEp-2 cells. These results may provide a basis for the clinical application of genistein and TSA in the treatment of laryngeal cancer.

\section{Acknowledgements}

This study was supported by grants from the Liaoning Provincial Department of Education Science Research Project (grant no. L2014299).

\section{References}

1. Miao S, Mao X, Pei R, Miao S, Xiang C, Lv Y, Yang X, Sun J, Jia S and Liu Y: Antitumor activity of polysaccharides from Lepista sordida against laryngocarcinoma in vitro and in vivo. Int J Biol Macromol 60: 235-240, 2013.

2. Xie J, Jin B, Li DW, Shen B, Cong N, Zhang TZ and Dong P: ABCG2 regulated by MAPK pathways is associated with cancer progression in laryngeal squamous cell carcinoma. Am J Cancer Res 4: 698-709, 2014.

3. Hoffman HT, Porter K, Karnell LH, Cooper JS, Weber RS, Langer CJ, Ang KK, Gay G, Stewart A and Robinson RA: Laryngeal cancer in the United States: Changes in demographics, patterns of care, and survival. Laryngoscope (Suppl 111) 116: 1-13, 2006.

4. Kraljevic Pavelic S, Cacev T and Kralj M: A dual role of p21waf1/ cipl gene in apoptosis of HEp-2 treated with cisplatin or methotrexate. Cancer Gene Ther 15: 576-590, 2008.

5. Li L, Jiang AC, Dong P, Wan Y and Yu ZW: The characteristics of Hep-2 cell with multiple drug resistance induced by Taxol. Otolaryngol Head Neck Surg 137: 659-664, 2007.

6. Groth-Pedersen L and Jäättelä M: Combating apoptosis and multidrug resistant cancers by targeting lysosomes. Cancer Lett 332 : 265-274, 2013.

7. Suzuki R, Kang Y, Li X, Roife D, Zhang R and Fleming JB: Genistein potentiates the antitumor effect of 5-Fluorouracil by inducing apoptosis and autophagy in human pancreatic cancer cells. Anticancer Res 34: 4685-4692, 2014.

8. Chen J, Lin C, Yong W, Ye Y and Huang Z: Calycosin and genistein induce apoptosis by inactivation of HOTAIR/p-Akt signaling pathway in human breast cancer MCF-7 cells. Cell Physiol Biochem 35: 722-728, 2015.

9. Beard N, Benghuzzi H, Tucci M and Cason Z: The effects of genistein concentrations on Hep-2 cellular function. Biomed Sci Instrum 41: 199-204, 2005.

10. Dynek JN and Vucic D: Antagonists of IAP proteins as cancer therapeutics. Cancer Lett 332: 206-214, 2013.

11. Li YL, Yang TS, Ruan WM, Cui W, Jin Y and Zou XM: Effect of trichostatin a on SGC-7901 gastric cancer cells. Int J Clin Exp Med 7: 1958-1966, 2014.

12. Han C, Gu H, Wang J, Lu W, Mei Y and Wu M: Regulation of L-threonine dehydrogenase in somatic cell reprogramming. Stem Cells 31: 953-965, 2013.

13. Ruan WM, Li YL, Nie G, Zhou WX and Zou XM: Differential expression of glycoprotein non-metastatic melanoma protein $\mathrm{B}$ (GPNMB) involved in trichostatin A-induced apoptosis in gastric cancer. Int J Clin Exp Med 7: 4857-4866, 2014.

14. Han C, Jin L, Mei Y and Wu M: Endoplasmic reticulum stress inhibits cell cycle progression via induction of p27 in melanoma cells. Cell Signal 25: 144-149, 2013.

15. Liu D, Yan L, Wang L, Tai W, Wang W and Yang C: Genistein enhances the effect of cisplatin on the inhibition of non-small cell lung cancer A549 cell growth in vitro and in vivo. Oncol Lett 8: 2806-2810, 2014.

16. Zhang LL, Li L, Wu DP, Fan JH, Li X, Wu KJ, Wang XY and He DL: A novel anti-cancer effect of genistein: Reversal of epithelial mesenchymal transition in prostate cancer cells. Acta Pharmacol Sin 29: 1060-1068, 2008

17. Wang X, Xu J, Wang H, Wu L, Yuan W, Du J and Cai S: Trichostatin A, a histone deacetylase inhibitor, reverses epithelial-mesenchymal transition in colorectal cancer SW480 and prostate cancer PC3 cells. Biochem Biophys Res Commun 456: 320-326, 2015.

18. Dai W, Wang F, He L, Lin C, Wu S, Chen P, Zhang Y, Shen M, Wu D, Wang C, et al: Genistein inhibits hepatocellular carcinoma cell migration by reversing the epithelial-mesenchymal transition: Partial mediation by the transcription factor NFAT1. Mol Carcinog 54: 301-311, 2015. 